\title{
An Evaluation of Quasi-Moment-Method Calibrated Pathloss Models for Benin City Nigeria
}

\author{
M. A. K. Adelabu, A. A. Ayorinde, H. A. Muhammed, F. O. Okewole, \\ A. I. Mowete* \\ Department of Electrical and Electronics Engineering, University of Lagos, Akoka, Lagos State, NIGERIA.
}

\begin{abstract}
This paper introduces the Quasi-Moment-Method (QMM) as a novel radiowave propagation pathloss model calibration tool, and evaluates its performance, using field measurement data from different cellular mobile communication network sites in Benin City, Nigeria. The QMM recognizes the suitability of component parameters of existing basic models for the definition of 'expansion' and 'testing functions' in a Galerkin approach, and simulations were carried out with the use of a FORTRAN program developed by the authors, supported by matrix inversion in the MATLAB environment. Computational results reveal that in terms of both Root Mean Square (RMS) and Mean Prediction (MP) errors, QMM-calibrated models performed much better than an 'optimum' model reported for the NIFOR (Benin City), by a recent publication. As a matter of fact, the QMM-calibrated COST231 (rural area) model recorded reductions in RMS error of between $31.5 \%$ and $71 \%$ compared with corresponding metrics due to the aforementioned 'optimum' model. The simulation results also revealed that of the five basic models (COST231-rural area and sub-urban city, ECC33 (medium and large sized cities), and Ericsson models) utilized as candidates, the two ECC33 models, whose performances were consistently comparable, represented the best models for QMM-model calibration in the Benin City environments investigated.
\end{abstract}

Keywords: moment method, pathloss modelling, ECC33, COST231-Hata, Ericsson

\section{INTRODUCTION}

Radiowave propagation pathloss 'calibration' (also known as 'tuning') may be described as that empirical process, with which a basic (or nominal) prediction model is adjusted to fit a profile prescribed by field measurements. Quite a few model tuning techniques have been reported in the open literature, with majority of them utilizing what is commonly referred to as the 'least square method'. Examples of these include the contribution by Chebil et al. [1], who, using measurement data for a sub-urban area in Kuala Lumpur (Malaysia), calibrated the Lee model, in a Least Square approach. The focus of the tuning was on two component parameters of the Lee model, namely, the pathloss exponent and the free space attenuation factor. RMSE values recorded by tuned model varied between $8.78 \mathrm{~dB}$ and 9.82dB. Dalela, Prasad, and Dalela [2], developed a linear iterative approach to the tuning of the COST231-Hata model. For calibration

\footnotetext{
* Corresponding author (Tel: +234(0) 813449 8678)

Email addresses: amadelabu@unilag.edu.ng (M. A. K. Adelabu), aayorinde@unilag.edu.ng (A. A. Ayorinde), hmuhammad@unilag.edu.ng (H. A. Muhammed), fokewole@unilag.edu.ng (F. O. Okewole), amowete@unilag.edu.ng (

A. I. Mowete)
}

purposes, the model focused on the two component parameters that are functions of the variable 'd'- radial distance of measurement point from transmitting antenna's location. Model performance evaluation was carried out through a comparison of predicted pathloss with measurement data from WiMAX network, and RMSE metrics recorded ranged between $6.6 \mathrm{~dB}$ and $9.2 \mathrm{~dB}$. The Least Square Algorithm utilized by Diawuo et al. [3], for the tuning of the Okumura-Hata model, is essentially a linear regression approach, in which two component parameters (the free space attenuation factor and the base station antenna height correction factor) are selected for 'optimization'. RMSE values in the range between $4.16 \mathrm{~dB}$ and $8.6 \mathrm{~dB}$ were recorded, when measurement values from a CDMA2000 network in Greater Accra, Ghana, were utilized for calibration. A Least Square Approximation approach was presented in the contribution by Deme, Dajab and Nyap [4], whose model is a quadratic in 'd'. According to the authors of [4], the coefficients of the quadratic expression represent the solution to an ill-defined system of 'normal equations' (Eqs. (3) of [4]); and the COST231-Hata model subsequently calibrated with measurement data from GSM900 network in Jos, Nigeria, recorded $5.1 \mathrm{~dB}$ as its RMSE metric. In addition to cal- 
ibrating the COST231-Hata Model, the publication by Salami et al. [5], also tuned the Stanford University Interim (SUI) model, using a linear regression technique with field measurement data obtained from Networks in Togo. The performances of both tuned models were not consistent, as RMSE values reported by the authors varied from as low as $1.523 \mathrm{~dB}$ in one case, to as high as $20.8009 \mathrm{~dB}$ in another. Unlike what obtained with the examples described in the foregoing discussions, Keawbunsong et al. [6], focused model calibration attention on a third generation terrestrial Digital Video broadcasting network. Their approach is also a least square linear regression method that incorporated a random error term. RMSE metrics reported in the paper ranged between $6.717 \mathrm{~dB}$ and $7.244 \mathrm{~dB}$, and may be regarded as consistent and satisfactory. Of particular interest to this paper is a recent publication by Omoze and Edeko [7], in which a least square approach, similar to that described in [2] was utilized for the tuning of the basic COST231-Hata model for rural areas, with field measurements taken from three GSM1800 networks in the NIFOR (Benin City) environment. Computational results (in dB) reported by the authors for (MPE, RMSE) were given as $(1.17,5.58)$ for 'Network A', $(2.20,7.16)$, for 'Network B', and $(6.21,10.78)$, for 'Network C'. This paper utilizes the same measurement data reported in [7] for the QMM-calibration of five different basic models, including the COST231Hata model for rural areas, considered by the authors of [7]. Computational (MPE, RMSE) results obtained for the QMM-calibrated COST231Hata (rural area) emerged as $(-0.22 \mathrm{~dB}, 3.80 \mathrm{~dB})$, for 'Network A', $(0.30 \mathrm{~dB}, 2.67 \mathrm{~dB})$, for 'Network B', and $(0.77 \mathrm{~dB}, 3.06 \mathrm{~dB})$, for 'Network C'. Although these metrics are clearly much better than those recorded by the optimum model of [7], even better metrics were obtained for the corresponding QMM-calibrated ECC-33 models. For example, in the case of the ECC-33 (medium city model), (MPE, RMSE) values were obtained as $(0.19 \mathrm{~dB}$, $1.19 \mathrm{~dB}),(0.03 \mathrm{~dB}, 2.04 \mathrm{~dB})$, and $(0.12 \mathrm{~dB}, 2.34 \mathrm{~dB})$, for networks 'A', 'B', and ' $\mathrm{C}$ ', respectively. Results concerning percentage contributions to net predicted pathloss by the component parameters of basic and corresponding QMM-calibrated models are also presented in the paper, to illustrate the model calibrating features of the Quasi-MomentMethod.

Section 2 of the paper describes the generic Quasi-Moment-Method problem formulation, and establishes equivalence between the Method of Moments as utilized for solution of electromagnetic field problems, and the QMM, as formulated here. Thereafter, the generic process is specialized for use with basic pathloss model calibration, using the basic models utilized in the paper as candidate examples. In section 3, the QMMmodel-calibration process is validated through a comparative evaluation of its performance with the optimized model reported in [7], for the NIFOR environment in Benin City. Further model evaluation is provided in the paper's section 4 , where outcomes of calibration with measurement data obtained by the authors for other areas of Benin City, are comprehensively discussed. In the paper's concluding remarks, it is noted that one key implication of the investigations reported here is that the fact that the performance of one basic (uncalibrated) model (in particular, the ECC33 model) is inferior to that of another should not form the basis for the choice of basic model to be optimized. Possible directions of future research are also identified, including the possibility of taking advantage of the inherent symmetry of the 'model-calibration matrix', to formulate and solve a pathloss eigenvalue problem.

\section{FORMULATION}

The formal approximation problem addressed by the Quasi-Moment-Method as utilized in this paper may be described as follows. Given a set $\left\{P_{l m e a}\left(d_{k}\right)\right\}_{k-1}^{N}$ of field measurement data, determine a function $P_{I Q}\left(d_{k}\right)$, such that weighted Euclidean semi-norm of the error function defined as

$$
\begin{aligned}
& \|\varepsilon\|=\left\|P_{\text {lmea }}-P_{l Q}\right\|= \\
& {\left[\sum_{k=1}^{N}\left(w_{k}\left|P_{l m e a}\left(d_{k}\right)-P_{l Q}\left(d_{k}\right)\right|^{2}\right)\right]^{\frac{1}{2}}}
\end{aligned}
$$

assumes its smallest possible value. In the formalism of the approximation problem so defined [8], the first step towards a solution to this problem is to prescribe the desired approximation function as a linear combination of known 'basis' or 'expansion' functions, according to

$$
P_{l Q}=c_{0} P_{l 0}+c_{1} P_{l 1}+\cdots c_{M} P_{l M},
$$

in which the set $\left\{P_{l m}\right\}_{m=0}^{M}$ is a set of known functions, and the set $\left\{c_{m}\right\}_{m=0}^{M=0}$ is a set of unknown coefficients (referred to in this paper as 'model calibration coefficients') to be determined. As described by Dahlquist and Bjork [8], the solution to this approximation problem begins with the definition of an inner (or scalar) product quantity for two functions $g_{1}\left(d_{k}\right)$ and $g_{2}\left(d_{k}\right)$ as

$$
\left\langle g_{1}, g_{2}\right\rangle=\sum_{k=1}^{N} w_{k} g_{1}\left(d_{k}\right) g_{2}\left(d_{k}\right)
$$

The function $w_{k}$, which appears in Eqns. (1) and (3) is a weighting function set equal to 1 everywhere in this paper. Next, a set, $\left\{P_{t}\right\}_{t=0}^{M}$, of 'testing functions' is chosen as being identical to set of 'basis' functions, and the inner product of each testing function and both sides of the equation

$$
P_{l Q}=c_{0} p_{l 0}+c_{1} p_{l 1}+\cdots c_{M} p_{l M}=P_{l m e a}
$$

is taken to yield the system of equations given as 


$$
\begin{aligned}
& \left\langle p_{l 0}, p_{l 0}\right\rangle c_{0}+\left\langle p_{l 0}, p_{l 1}\right\rangle c_{1}+\cdots\left\langle p_{l 0}, p_{l M}\right\rangle c_{m}= \\
& \left\langle p_{l 0}, p_{l m e a}\right\rangle \\
& \vdots \\
& \left\langle p_{l M}, p_{l 0}\right\rangle c_{0}+\left\langle p_{l M}, p_{l 1}\right\rangle c_{1}+\cdots\left\langle p_{l M}, p_{l M}\right\rangle c_{m}= \\
& \left\langle p_{l M}, p_{l m e a}\right\rangle
\end{aligned}
$$

Clearly, Eq. (5) admits the matrix description given as

$$
[\Phi](C)=(\Pi),
$$

in which entries into the column vector symbolized by $(C)$ are the unknown coefficients, to be determined. Because entries into the matrix $[\Phi]$ are sums of products of the known 'basis' and 'testing' functions, and since the column vector (П) is populated by products of known field measurement data and 'testing' functions, the desired 'model calibration coefficients' are obtained as the solution to the approximation problem, through the matrix operations of inversion and multiplication, according to

$$
(C)=[\Phi]^{-1}(\Pi) \text {. }
$$

The process described here for the modelcalibration technique is similar to the circuitgeometric Method of Moments (MoM), originally developed by Harrington [9, 10], for the solution of electromagnetic field problems.

There are two major requirements for the successful use of the method of moments (MoM); and these are [10]:

(i) choosing expansion functions that are linearly independent, and which are such that a linear combination of the type specified by Eq. (2) can be expected to provide a reasonable approximation, and

(ii) choice of linearly independent 'testing' functions in a manner that ensures that the inner product quantities on the right-hand-side of Eq. (5) depend only on the relatively independent properties of ' $P_{\text {lmea }}$ ', in the case of interest here.

The particular choice of identical 'expansion' and 'testing' functions is referred to as the 'Galerkin' approach [10], which has been rigorously shown [11], to represent an equivalent of the Rayleigh-Ritz variational technique. In terms of linear spaces, the particular case of the Galerkin method represents some projection of the approximate solution on the desired solution in an errorminimizing process, [9]. Furthermore, because the error and projection are orthogonal functions, the process is a second order. The foregoing discussions concerning the MoM have their equivalents in the QMM. In particular, the linear independence of both the 'expansion' and 'testing' functions is also a requirement, though in this case, the purpose is to ensure the uniqueness of the solution[8]. As also noted in [8], the use of Euclidean semi-norm admits the physical interpretation that the solution to the approximation problem may be regarded as a generalization of the well-known fact of two-and three-dimensional geometry, that the shortest distance between a point and a linear subspace is the length of the vector connecting the point to a point located on the subspace, along a perpendicular. And hence, as is the case with MoM, the error in QMM is orthogonal to the subspace spanned by the expansion functions [8].

Since the basic pathloss models were particularly developed for the purposes of pathloss prediction, and because their component parameters are inherently linearly independent (as formally shown in section 2.1), the component parameters are recognized here as representing natural choices for 'expansion' (and 'testing') functions. This observation forms the basis of the specialization process described in the following section.

\subsection{Specialization to Basic Pathloss Model Calibration}

The specialization of the QMM technique as formulated in $\S 2$, to the basic pathloss model calibration of interest here is essentially that of prescribing 'basis' and 'testing' functions according to Eq. (2). These 'basis' and 'testing' functions, for the five candidate models utilized in this paper are identified by the following expressions.

For the basic Ericsson pathloss model, [12], the desired equation is

$$
\begin{aligned}
P_{l Q-E r i c}= & c_{0}(36.2)+c_{1}\left(30.2 \log _{10} d\right) \\
& +c_{2}\left(-12 \log _{10} h_{t e}\right) \\
& +c_{3}\left(0.1 \log _{10} d \log _{10} h_{t e}\right) \\
& +c_{4}\left(-3.2 \log _{10}\left(11.75 h_{r e}\right)^{2}\right) \\
& +c_{5}(g(f))
\end{aligned}
$$

whereas, they are given by

$$
\begin{aligned}
& G_{f r Q}=c_{0}(92.4)+c_{1}\left(20 \log _{10} d\right)+c_{2}\left(20 \log _{10} f\right) \\
& G_{b m Q}=c_{3}(20.41)+c_{4}\left(9.83 \log _{10} d\right) \\
& +c_{5}\left(\left[7.894+9.56 \log _{10} f\right] \log _{10} f\right) \\
& G_{t e Q}=c_{6}\left(13.958 \log _{10}\left(\frac{h_{t e}}{200}\right)\right)+ \\
& c_{7}\left(5.8 \log _{10}\left(\frac{h_{t e}}{200}\right)\left(\log _{10} d\right)^{2}\right)
\end{aligned}
$$

for both ECC33 large and medium city models, provided that it is understood that the associated basic models are given by, [7],

$$
P_{l B-E C C}=G_{f r}+G_{b m}-G_{h t e}-G_{h r e}
$$

The terms on the right-hand member of Eq. (9d) as component parameters of the basic ECC-33 
pathloss models, represent 'free space attenuation', 'basic median pathloss', 'transmitter antenna height correction factor', and 'receiver antenna height correction factor', respectively. And as suggested by Eqs. (9a), (9b), and (9c), the first three terms are common to the large-city and medium city models. The 'receiver antenna height correction factor' however, has different expressions for the aforementioned models; thus, we find that [7],

$$
\begin{aligned}
G_{h r e Q}= & c_{8}\left(42.57\left(\log _{10} h_{r e}-0.585\right)\right) \\
& +c_{9}\left(1 3 . 7 \operatorname { l o g } _ { 1 0 } f \left(\log _{10} h_{r e}\right.\right. \\
& -0.585))
\end{aligned}
$$

in the case of medium cities; and

$$
G_{h r e Q}=c_{8}\left(0.759 h_{r e}\right)+c_{9}(-1.862)
$$

for large cities. Expressions for the calibrated COST231-Hata models will, for terms common to both 'rural area' and 'sub-urban city', [7], assume the form

$$
\begin{aligned}
P_{l Q-C 231}= & c_{0}(46.33)+c_{1}\left(33.9 \log _{10} f\right) \\
& +c_{2}\left(-13.82 \log _{10} h_{t e}\right) \\
& +c_{3}\left(44.9 \log _{10} d\right) \\
& +c_{4} h_{t e}\left(-6.55 \log _{10} d\right)
\end{aligned}
$$

Terms additional to Eq. (11) represent correction factors for mobile station antenna height, and are given by, [7, 10],

$$
\alpha\left(h_{r e}\right)_{Q}=c_{5}\left(h_{r e}\left(0.7-1.1 \log _{10} f\right)\right)+c_{6}\left(1.56 \log _{10} f-0.8\right)
$$

in the case of 'rural areas', and by

$$
\alpha\left(h_{r e}\right)_{Q}=c_{5}\left(-3.2 \log _{10}\left(11.75 h_{r e}\right)^{2}+4.97\right)
$$

for sub-urban cities.

It is matter of interest to now formally demonstrate that the expansion and testing functions prescribed by the candidate models do indeed, meet the requirements of being linearly independent. In that connection, and as shown by Bostan and Dumas, [13], the functions will be linearly independent, if the Wronskian associated with them is non-zero. The Ericsson and COST231-Hata models, for given antenna heights and operating frequencies, essentially, as functions of ' $d$ ', reduce, to forms given as

$$
f_{1}(d)=k_{1}+k_{2} \log _{10} d
$$

in which $\mathrm{k}_{1}$ and $\mathrm{k}_{2}$ are constants, and whose Wronskian is readily evaluated as

$$
W\left(f_{1}\right)=\operatorname{det}\left(\left[\begin{array}{cc}
k_{1} & k_{2} \log _{10} d \\
0 & k_{2} / d \log _{e} 10
\end{array}\right]\right)=\frac{2 k_{1} k_{2} k_{3}}{\left(d \log _{e} 10\right)^{3}}
$$

and is clearly a non-zero quantity, for all finite values of 'd'. Similarly, the ECC-33 models, for given antenna heights and operating frequency, can be written as

$$
f_{2}(d)=k_{1}+k_{2} \log _{10} d+k_{1}+k_{3}\left(\log _{10} d\right)^{2}
$$

so that

$W\left(f_{2}\right)=$

$\operatorname{det}\left(\left[\begin{array}{cll}k_{1} & k_{2} \log _{10} d & k_{3}\left(\log _{10} d\right)^{2} \\ 0 & k_{2} / d \log _{e} 10 & 2 k_{3} \log _{10} d / d \log _{e} 10 \\ 0 & -2 k_{2} / d^{2} \log _{e} 10 & \frac{2 k_{3}}{\left(\log _{e} 10\right)^{2}} \\ & =\frac{2 k_{2} k_{3}}{\left.\left(d \log _{e} 10\right)\left(\log _{10} d\right)-1\right)}\end{array}\right]\right)$

which is also non-zero, for finite values of 'd'.

\section{MODEL VALIDATION}

In order to establish the validity and efficiency of QMM-model calibration approach, the technique is utilized for the calibration of the five candidate models, as described in $\S 2$, using measurement data available (through the commercial graph digitizer 'GETDATA'- https://getdatagraph-digitizr.com) from Figs. 6, 7, and 8, of [7], for the networks identified as 'A', 'B', and ' $\mathrm{C}$ ', respectively, by that publication.

\subsection{Comparative Performance Evaluation}

Solutions to the approximation problem associated with the calibration of the candidate models with the use of measurement data extracted from Fig. 6 of [7], are given, in terms of their corresponding 'model calibration coefficients' as follows:

$$
\begin{aligned}
(C)_{C O S T-R U R}= & (1.6409-0.6559-1.5139 \\
& 4.404317 .3453-25.4938 \\
& 2.1919)^{T} \\
& \\
(C)_{C O S T-S U B}= & (3.2873-0.3792-21619 \\
& 4.041515 .7930 \\
& 21.6062)^{T} \\
(C)_{E C C-L}=\quad(0.86190 .48743 .5312 & 1.14531 .42943 .7657
\end{aligned}
$$

3.42143 .181310 .0741

$$
2.4656)^{T}
$$

$$
\begin{aligned}
(C)_{E C C-M}= & (1.56740 .45609 .1059 \\
& 1.45911 .4918-5.1626 \\
& -3.20223 .1808-1.3590 b \\
& 3.4806)^{T}
\end{aligned}
$$



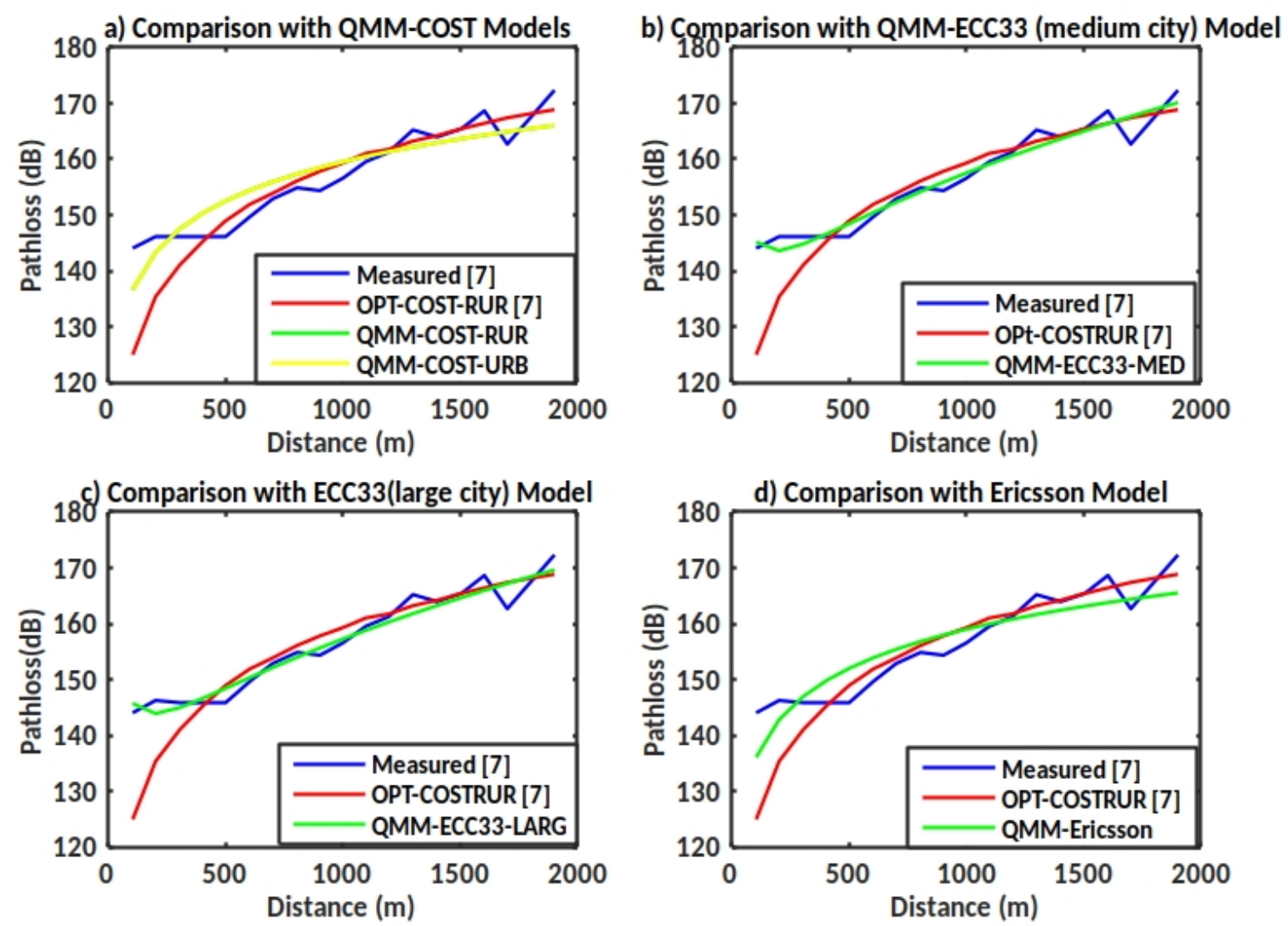

Figure 1: Profiles of predicted and measured pathloss for 'Network A' of [7].

$$
\begin{aligned}
(C)_{E R I C}= & (-2.34550 .5594-11.0931 \\
& -7.1912-21.0952-1.2996)(15 \mathrm{e})
\end{aligned}
$$

Figure 1 displays the predicted pathloss profiles due to QMM calibrated models prescribed by the solutions of Eq. (15), compared with measurement and simulation from Fig. 6 of [7].

Corresponding results for 'Network B' of [7] emerge as

$$
\begin{aligned}
(C)_{C O S T-R U R}= & (1.6409-0.6559-1.5139 \\
& 4.404317 .3453-25.4938 \\
& 2.1919)^{T} \\
& \\
(C)_{C O S T-S U B}= & (3.2873-0.3792-21619 \\
& 4.041515 .7930 \\
& 21.6062)^{T} \\
(C)_{E C C-L}= & (0.86190 .48743 .5312 \\
& 1.14531 .42943 .7657 \\
& 3.42143 .181310 .0741 \\
2.4656)^{T} & (16) \\
= & 1.56740 .45609 .1059 \\
(C)_{E C C-M}= & 3.20223 .1808-1.3590 b \\
& 3.4806)^{T}
\end{aligned}
$$

$$
\begin{aligned}
(C)_{E R I C}= & (-2.34550 .5594-11.0931 \\
& -7.1912-21.0952 \\
& -1.2996)^{T}
\end{aligned}
$$

With these results as solutions to the QMMmodel calibration problem concerning Fig. 7 of [7], the pathloss profiles predicted by the five calibrated basic candidate models, as compared with measurement and simulations results available for 'Network B' of [7] are as shown in Fig. 2.

Finally, the five candidate basic models were calibrated with the use of measurement results extracted from Fig. 8 of [7], to give the model calibration coefficients of Eq. (17) and the corresponding pathloss profiles of Fig. 3 .

The pathloss profiles of Figs. 1, 2, and 3, as well as the associated statistical performance metrics of MPE and RMSE displayed in Table 1 very clearly reveal that all QMM-calibrated models recorded much better performances than the 'optimum' model utilized in [7]. Accordingly, the validity of the QMM as a basic pathloss calibration tool is firmly established, and the efficiency of the remarkably easy to implement algorithm is also demonstrated by these results.

\subsection{Model Prediction Characteristics}

As mentioned earlier, and according to theorem 4.2.5 of Dahlquist and Bjorck, [8], the solution to the QMM-calibration problem is unique, 

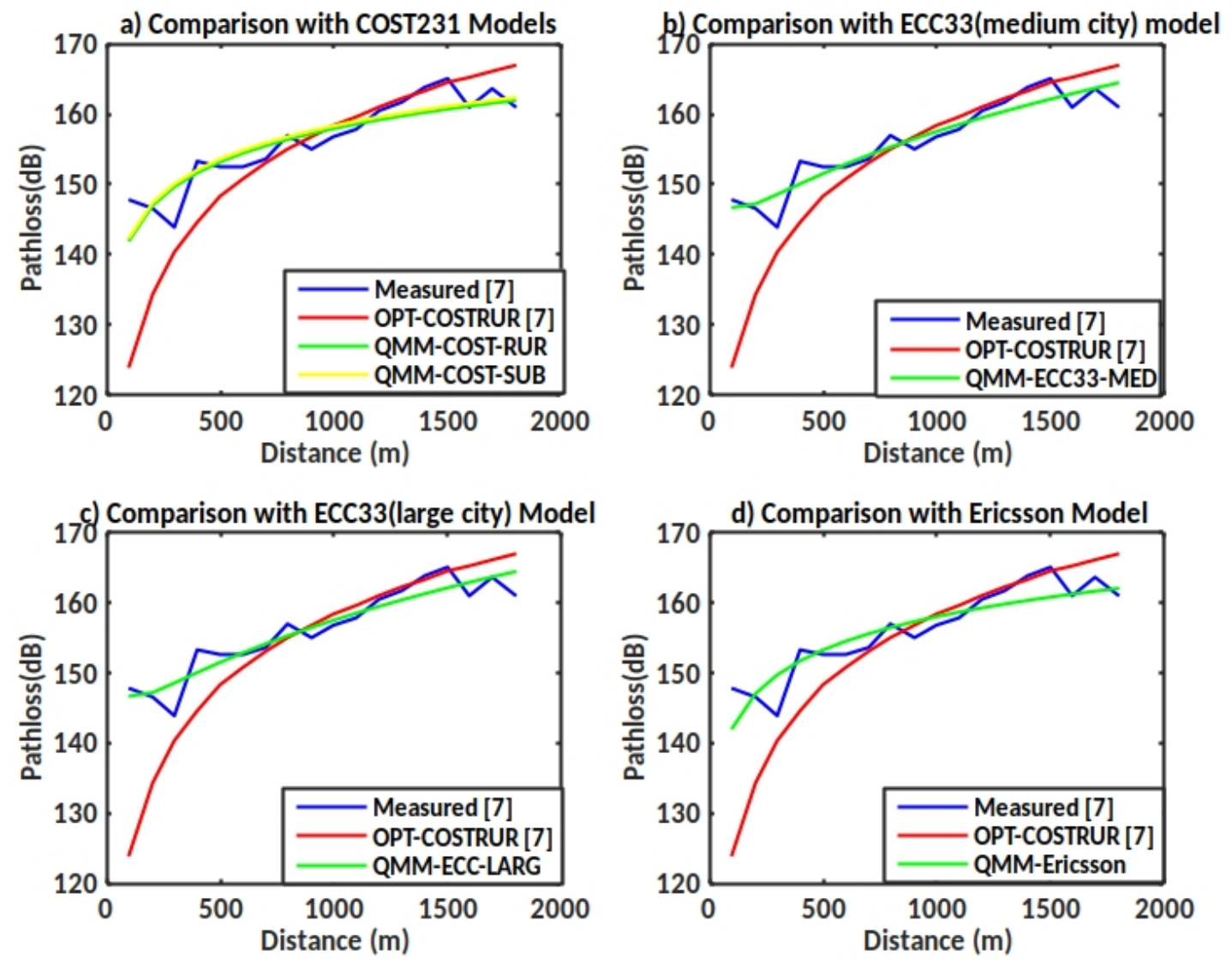

Figure 2: Profiles of predicted and measured pathloss for 'Network B' of [7].

a) Comparison with COST231 Models
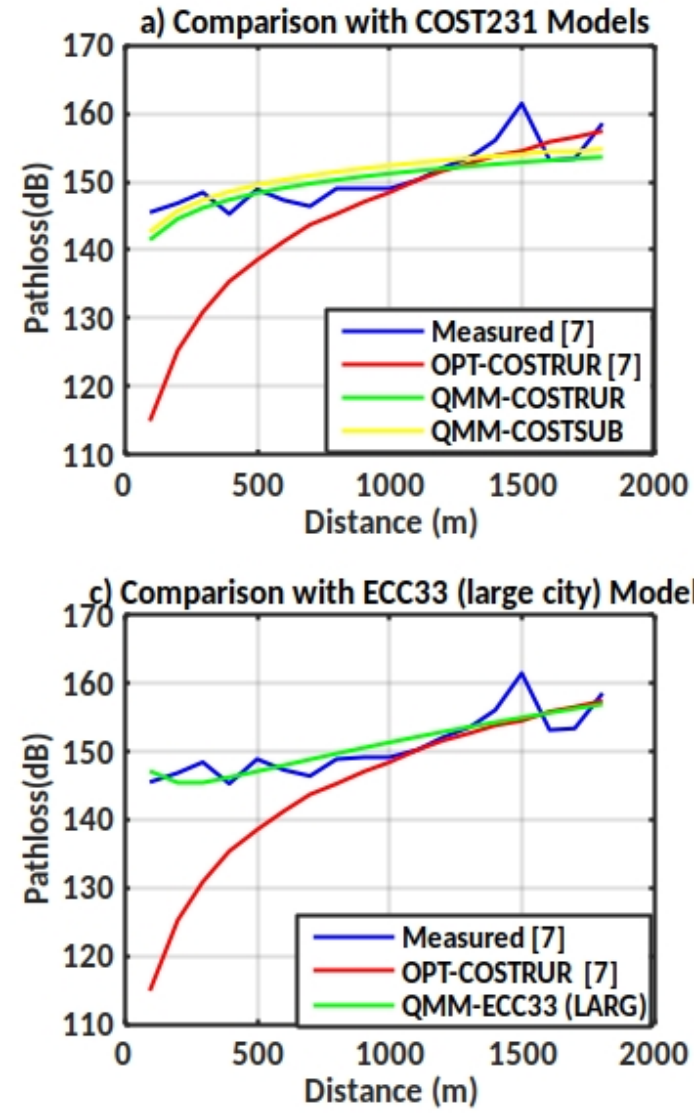
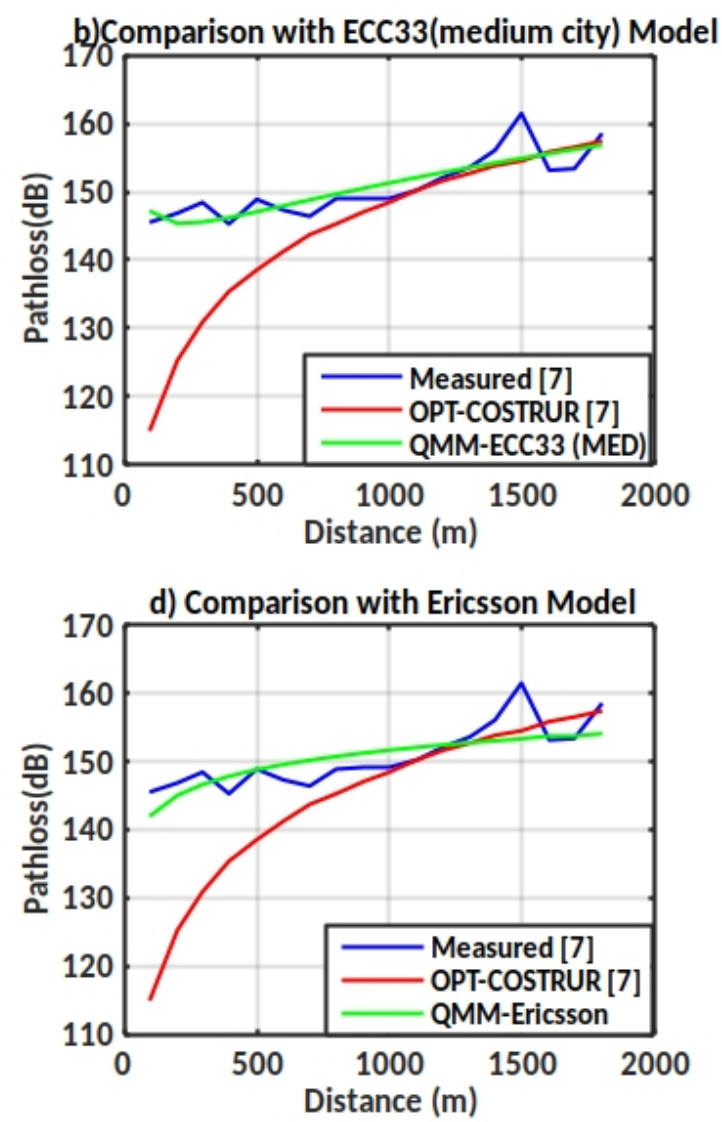

Figure 3: Profiles of predicted and measured pathloss for 'Network C' of [7]. 
Table 1: Statistical performance metrics for the pathloss profiles.

\begin{tabular}{lllllll}
\hline \multirow{2}{*}{ Model/Metric } & \multicolumn{3}{c}{ Network A [7] } & \multicolumn{3}{c}{ Locations } \\
& Network B [7] & \multicolumn{2}{c}{ Network C [7] } \\
& RMSE & MPE & RMSE & MPE & RMSE & MPE \\
\hline COST231(RUR)[7] & 5.58 & 1.17 & 7.16 & 2.26 & 10.78 & 6.21 \\
COST231(RUR)-QMM & 3.80 & -0.22 & 2.67 & 0.30 & 3.06 & 0.77 \\
COST231 (S-URB) QMM & 3.80 & -0.22 & 2.65 & -0.09 & 2.99 & -0.41 \\
ECC (MEDIUM)-QMM & 1.91 & 0.19 & 2.04 & 0.03 & 2.34 & -0.12 \\
ECC (LARGE) -QMM & 1.89 & 0.03 & 2.04 & 0.01 & 2.33 & -0.08 \\
ERICSSON QMM & 3.80 & 0.29 & 2.66 & 0.24 & 2.98 & 0.30 \\
\hline
\end{tabular}

provided that the 'basis' functions of Eq. (2) are linearly independent. It is of interest therefore, to examine how these basis functions contribute to net pathloss, before, and after QMM calibration. Using computational data for the COST-231 Hata models calibrated with measurement data for 'Network B' of [7] as an illustrative example, the profiles of Fig. 4 describe contributions (as percentages of the net) to net predicted pathloss by component parameters of the basic and calibrated models.

$$
\begin{aligned}
(C)_{\text {COST-RUR }}= & (2.1103-0.9122-2.7253 \\
& 0.64761 .8688-11.9619 \\
& 9.6920)^{T} \\
(C)_{\text {COST-SUB }}= & (10.8556 n-3.2603-0.4109 \\
& -43.4135-186.6616 \\
& 113.7860)^{T}
\end{aligned}
$$

$$
\begin{aligned}
(C)_{E C C-L}= & (1.26602 .89451 .4465 \\
& 0.8011-4.04470 .5429 \\
& 0.68353 .40686 .5999 \\
& 5.3496)^{T}
\end{aligned}
$$

$$
\begin{aligned}
(C)_{E C C-M}= & (1.82131 .06534 .6704 \\
& 1.3425-0.3122-4.6266 \\
& -4.29523 .4063-1.1069 \\
& 3.6607)^{T}
\end{aligned}
$$

$$
\begin{aligned}
(C)_{E R I C}= & (-2.50830 .2963-6.7771 \\
& 3.2076-8.2343 \\
& 0.4551)^{T}
\end{aligned}
$$

Computational results, from which the profiles of Fig. 4 derive are shown in Tables 2 and 3 below. It is evident from the graphical illustrations and the numerical results that QMM-calibration informed by field measurements, considerably modifies contributions to net pathloss, by the component parameters of the models.

Two examples should suffice to illustrate this observation. In the basic model for rural areas, component parameter with $\log _{10} f$ as a factor contributed about $46.5 \%$ of the net predicted pathloss, close to the transmitter, and the contributions changed steadily to get to $8.04 \%$ at the far end. After calibration, this component parameter's contributions ranged from $-142.07 \%$ at the near end to $31.2 \%$ at the far end. On the other hand, contributions to this basic model's predicted pathloss by the parameter with $\log _{10} d$ as a factor varied from between $111 \%$ and $76 \%$ , and this, after QMM-calibration changed to between $-51 \%$ and $-44.68 \%$, as can be seen from Fig. 4 and Table 2. These same parameters, as seen from Table 3 for the sub-urban city case, recorded values of

$(\{76.77 \%, 58.8 \%\}-$ basic $\operatorname{vs}(\{-29.4 \%, 25.77 \%\}-$ QMM)' and $(\{-31.8 \%, 0.14 \%\}-$ basic $\operatorname{vs}(\{-130.04 \%, 26.67 \%\}-$ QMM)' for the parameters including $\log _{10} f$ and $\log _{10} d$ as factors, respectively. It should be remarked that these contributions are moderated by those from other component parameters, to give the net predicted pathloss.

\section{FURTHER MODEL PERFORMANCE EVAL- UATION}

In furtherance of the paper's main objective of characterizing QMM-pathloss model calibration performance in Benin City, the five candidate basic models earlier defined in Section 2, were subjected to QMM-calibration; in this case, using measurement results acquired in the field by the authors, [14]. Details of the measurement procedures (including design, instrumentation, and post-measurement processing) are available in [14]. In this section, outcomes of the QMMcalibration due to the four representative transmitters identified by the relevant site details of Table 4, are presented.

For the computational results described in this section, receiver height $\left(h_{r e}\right)$ is set at the default value of $1.5 \mathrm{~m}$, whilst $h_{t e}=45 \mathrm{~m}$ for the $1800 \mathrm{MHz}$ and $900 \mathrm{MHz}$ transmitters, respectively.

The pathloss profiles of Figs. 5 and 6 are for the two $1800 \mathrm{MHz}$ transmitters, whilst those of Figs. 7 and 8 are for the two $900 \mathrm{MHz}$ transmitters listed in Table 4, respectively. Statistical performance metrics for the calibrated models are displayed in Table 5.

From Table 5, it is readily seen that the best performing calibrated models are the ECC-33 models, whose statistical performance metrics are comparable. In the case of the $900 \mathrm{MHz}$ networks, for example, both calibrated models 

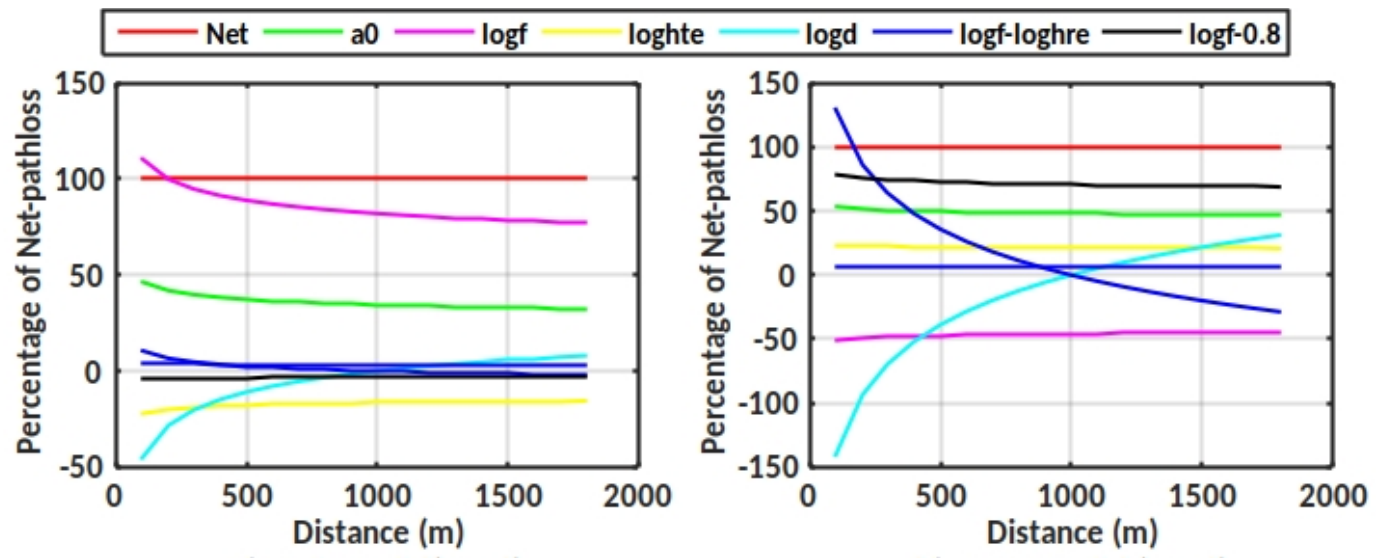

a) Basic Model(Rural)

b) QMM Model (Rural)

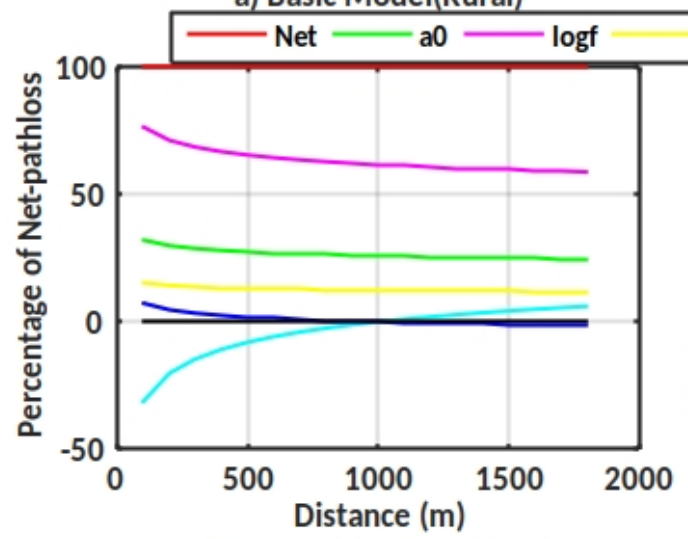

c) Basic Model (sub-urban)

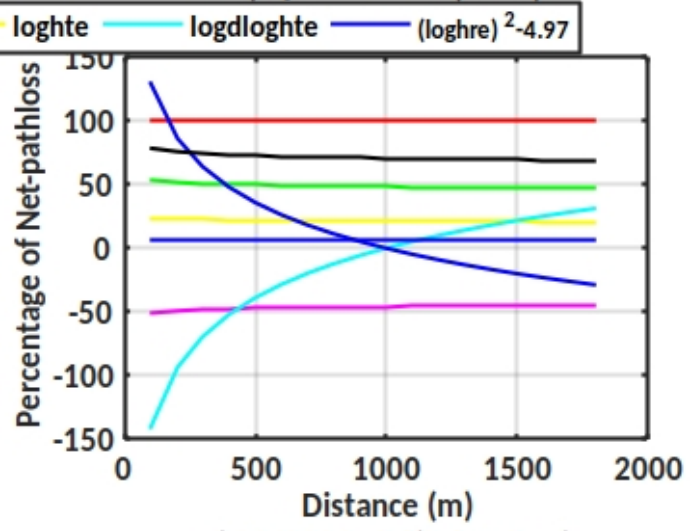

c) QMM Model(Sub-urban)

Figure 4: Percentage contributions to net pathloss by component parameters of basic and QMM-Calibrated COST231-Hata Models (Calibration with Fig. 7 of [7])
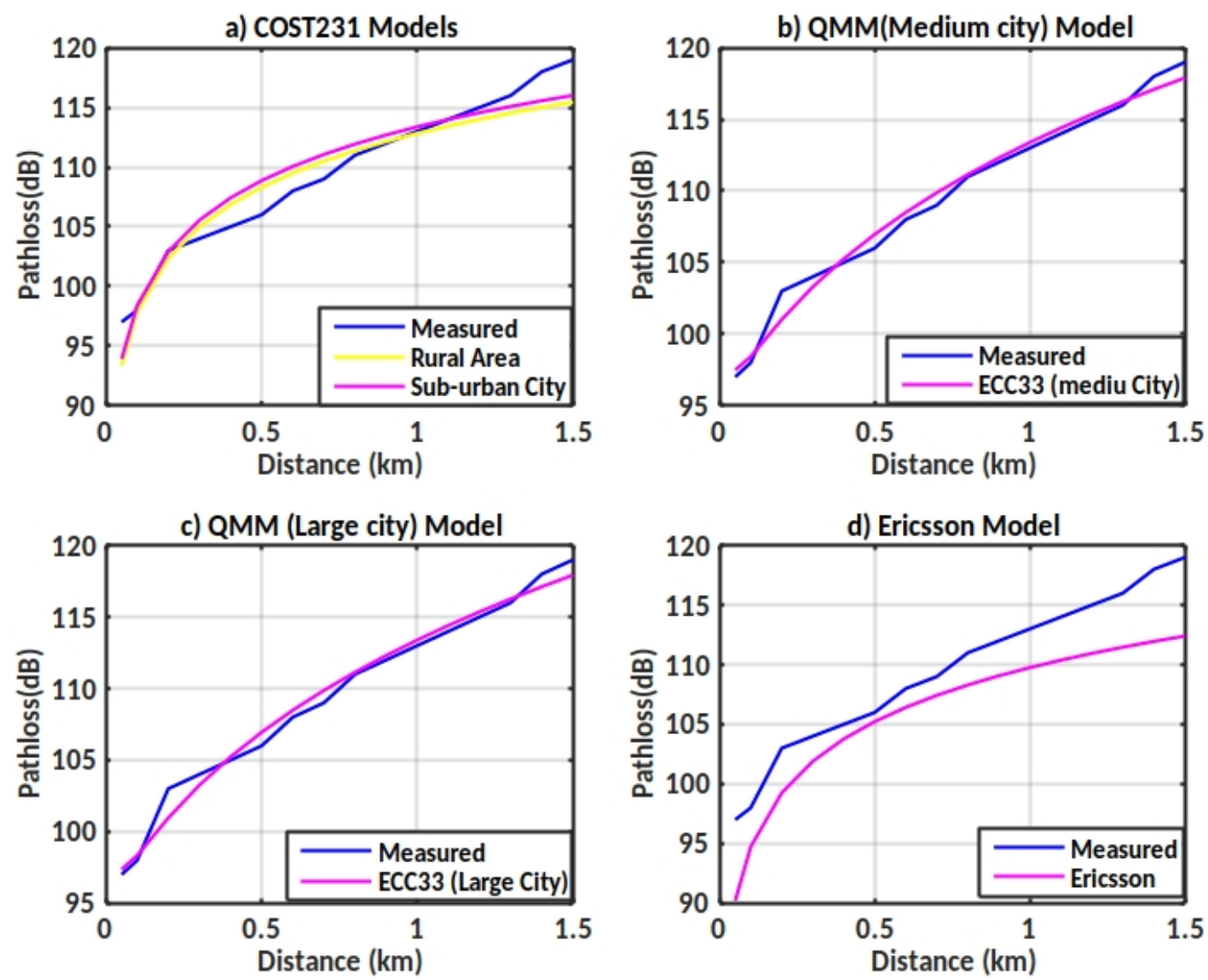

Figure 5: Comparison of pathloss predicted by QMM-models with measured data (ED0068). 
Table 2: Percentage contributions to net predicted pathloss by component parameters of the COST231-Hata (rural area) basic and QMM-calibrated models.

\begin{tabular}{|c|c|c|c|c|c|c|c|c|c|c|c|c|c|c|}
\hline \multicolumn{15}{|c|}{ Model Component Parameters } \\
\hline & $a_{0}$ & $6.33)$ & 33.91 & $g_{10} f$ & -13.821 & $g_{10} h_{t e}$ & 44.91 & $\operatorname{og}_{10} d$ & $-6.551 \mathrm{o}$ & $\mathrm{dlog}_{10} h_{t e}$ & (1.1log & f-0.7) $\boldsymbol{h}_{t e}$ & $1.56 \mathrm{lo}$ & $10 \mathrm{f}-0.8$ \\
\hline $\begin{array}{l}\text { d } \\
(\mathrm{km})\end{array}$ & $\begin{array}{c}\text { Basic } \\
(\%)\end{array}$ & $\underset{(\%)}{\mathbf{Q M M}}$ & $\underset{(\%)}{\text { Basic }}$ & $\underset{(\%)}{\text { QMM }}$ & $\underset{(\%)}{\text { Basic }}$ & $\underset{(\%)}{\mathbf{Q M M}}$ & $\underset{(\%)}{\text { Basic }}$ & $\underset{(\%)}{\mathbf{Q M M}}$ & $\underset{(\%)}{\text { Basic }}$ & $\underset{(\%)}{\mathbf{Q M M}}$ & $\underset{(\%)}{\text { Basic }}$ & $\underset{(\%)}{\mathbf{Q M M}}$ & $\underset{(\%)}{\text { Basic }}$ & $\underset{(\%)}{\mathbf{Q M M}}$ \\
\hline 0.10 & 46.60 & 53.58 & 111.00 & -51.01 & -22.27 & 23.62 & -46.05 & -142.10 & 10.76 & 130.79 & -4.35 & 78.51 & 4.30 & 6.61 \\
\hline 0.20 & 41.90 & 51.71 & 99.79 & -49.24 & -20.02 & 22.80 & -28.23 & 93.51 & 6.60 & 86.07 & -3.91 & 75.79 & 3.87 & 6.38 \\
\hline 0.30 & 39.81 & 50.80 & 94.82 & -48.37 & -19.02 & 22.40 & -20.32 & -69.60 & 4.' & 64.06 & -3.71 & 74.44 & 3.68 & 6.27 \\
\hline 0.40 & 38.37 & 50.13 & 91.40 & -47.73 & -18.34 & 22.10 & -14.87 & -52.14 & 3 & 47.99 & -3.58 & 73 & 3.54 & 6.18 \\
\hline 0.50 & 37.33 & 49.62 & 88.91 & -47.25 & -17.84 & 21.88 & -10.92 & -38.96 & 2. & 35.86 & -3.48 & 72.72 & 3.45 & 6.12 \\
\hline 0.60 & 36.52 & 49.22 & 86.98 & -46.86 & -17.45 & 21.70 & -7.85 & -28.40 & $1 . \varepsilon$ & 26.14 & -3.41 & 72.13 & 3.37 & 6.07 \\
\hline 0.70 & 35.86 & 48.88 & 85.42 & -46.54 & -17.14 & 21.55 & -5.36 & -19.63 & & 18.07 & -3.34 & 71.64 & 3.31 & 6.03 \\
\hline 0.80 & 35.34 & 48.61 & 84.18 & -46.28 & -16.89 & 21.43 & -3.3 & -12.50 & & 11.50 & -3.30 & 71.24 & 3.26 & 6.00 \\
\hline 0.90 & 34.84 & 48.35 & 82.99 & -46.03 & -16.65 & 21.32 & -1.50 & -5.59 & 0. & 5.15 & -3.25 & 70.85 & 3.22 & 5.96 \\
\hline 1.00 & 34.45 & 48.14 & 82.06 & -45.83 & -16.46 & 21.22 & -0.03 & -0.10 & 0. & 0.09 & -3.21 & 70.54 & 3.18 & 5.94 \\
\hline 110 & 34.09 & 47.94 & 81.19 & -45.64 & -16.29 & 21.14 & 1.36 & 5.12 & & -4.71 & -3.18 & & 3.15 & 5.91 \\
\hline 1.20 & 33.76 & 47.76 & 80.42 & -45.47 & -16.13 & 21.06 & 2.59 & 9.84 & & -9.05 & -3.15 & 69.98 & 3.12 & 5.89 \\
\hline 1.30 & 33.47 & 47.59 & 79.72 & -45.31 & -15.99 & 20.98 & 3.71 & 14.14 & 0 . & 13.02 & -3.12 & 69.74 & 3.09 & 5.87 \\
\hline 1.40 & 33.20 & 47.44 & 79.08 & -45.17 & -15.87 & 20.92 & 4.72 & 18.10 & & 16.66 & -3.10 & & 3.07 & 5.85 \\
\hline 1.50 & 32.95 & 47.30 & 78.50 & -45.03 & -15.75 & 20.85 & 5.65 & 21.76 & & 20.03 & -3.07 & 69.31 & 3.04 & 5.83 \\
\hline 1.60 & 32.74 & 47.17 & 77.98 & -44.91 & -15.65 & 20.80 & 6.46 & 24.99 & & 23.00 & -3.05 & 69.13 & 3.02 & 5.82 \\
\hline 1.70 & 32.53 & 47.05 & 77.48 & -44.80 & -15.55 & 20.75 & 7.26 & 28.18 & 1. & 25.94 & -3.03 & 68.95 & 3.00 & 5.80 \\
\hline 1.80 & 32.32 & 46.93 & 76.99 & -44.68 & -15.45 & 20.69 & 8.04 & 31.32 & 1.88 & 28.83 & -3.01 & 68.78 & 2.98 & 5.79 \\
\hline
\end{tabular}

Table 3: Percentage contributions to net predicted pathloss by component parameters of the COST231-Hata (sub-urban city) basic and QMM-calibrated models.

\begin{tabular}{|c|c|c|c|c|c|c|c|c|c|c|c|c|}
\hline \multicolumn{13}{|c|}{ Model Component Parameters } \\
\hline & $a_{0}(4$ & 6.33) & 33.91 & $g_{10} f$ & $-13.821 \mathrm{c}$ & $g_{10} h_{t e}$ & 44.91 & $\operatorname{og}_{10} d$ & $-6.551 \mathrm{o}$ & ${ }_{0} \operatorname{dlog}_{10} h_{t e}$ & $-3.2(\log$ & (11.75h) \\
\hline$\underset{(\mathrm{km})}{\mathbf{d}}$ & $\underset{(\%)}{\text { Basic }}$ & $\underset{(\%)}{\mathbf{Q M M}}$ & $\underset{(\%)}{\text { Basic }}$ & $\underset{(\%)}{\mathbf{Q M M}}$ & $\underset{(\%)}{\text { Basic }}$ & $\underset{(\%)}{\mathbf{Q M M}}$ & $\underset{(\%)}{\text { Basic }}$ & $\underset{(\%)}{\text { QMM }}$ & $\underset{(\%)}{\text { Basic }}$ & $\underset{(\%)}{\mathbf{Q M M}}$ & $\underset{(\%)}{\text { Basic }}$ & $\underset{(\%)}{\mathbf{Q M M}}$ \\
\hline 0.10 & 32.23 & 107.03 & 76.77 & -29.41 & 15.40 & 33.64 & -31.85 & -130.04 & 7.44 & 118.76 & 0.0006 & 0.01 \\
\hline 0.20 & 29.91 & 103.33 & 71.24 & -28.39 & 14.29 & 32.47 & -20.15 & -85.58 & 4.71 & 78.16 & 0.0006 & 0.01 \\
\hline 0.30 & 28.83 & 101.50 & 68.67 & -27.89 & 13.78 & 31.90 & -14.72 & -63.70 & 3.44 & 58.18 & 0.0006 & 0.01 \\
\hline 0.40 & 28.07 & 100.17 & 66.85 & -27.52 & 13.41 & 31.48 & -10.88 & -47.72 & 2.54 & 43.58 & 0.0006 & 0.01 \\
\hline 0.50 & 27.51 & 99.16 & 65.51 & -27.25 & 13.14 & 31.16 & -8.04 & -35.66 & 1.88 & 32.56 & 0.0005 & 0.01 \\
\hline 0.60 & 27.06 & 98.35 & 64.46 & -27.02 & 12.93 & 30.91 & -5.82 & -26.00 & 1.36 & 23.74 & 0.0005 & 0.01 \\
\hline 0.70 & 26.70 & 97.68 & 63.60 & -26.84 & 12.76 & 30.70 & -3.99 & -17.97 & 0.93 & 16.41 & 0.0005 & 0.01 \\
\hline 0.80 & 26.41 & 97.14 & 62.91 & -26.69 & 12.62 & 30.53 & -2.53 & -11.44 & 0.59 & 10.45 & 0.0005 & 0.01 \\
\hline 0.90 & 26.13 & 96.61 & 62.24 & -26.55 & 12.49 & 30.36 & -1.13 & -5.12 & 0.26 & 4.68 & 0.0005 & 0.01 \\
\hline 1.00 & 25.91 & 96.19 & 61.72 & -26.43 & 12.38 & 30.23 & -0.02 & -0.09 & 0.00 & 0.08 & 0.0005 & 0.01 \\
\hline 1.10 & 25.71 & 95.80 & 61.23 & -26.32 & 12.28 & 30.11 & 1.02 & 4.68 & -0.24 & -4.28 & 0.0005 & 0.01 \\
\hline 1.20 & 25.52 & 95.43 & 60.78 & -26.22 & 12.20 & 29.99 & 1.96 & 9.00 & -0.46 & -8.22 & 0.0005 & 0.01 \\
\hline 1.30 & 25.35 & 95.11 & 60.38 & -26.13 & 12.11 & 29.89 & 2.81 & 12.95 & -0.66 & -11.82 & 0.0005 & 0.01 \\
\hline 1.40 & 25.20 & 94.80 & 60.02 & -26.05 & 12.04 & 29.80 & 3.58 & 16.57 & -0.84 & -15.13 & 0.0005 & 0.01 \\
\hline 1.50 & 25.06 & 94.52 & 59.68 & -25.97 & 11.97 & 29.71 & 4.29 & 19.92 & -1.00 & -18.19 & 0.0005 & 0.01 \\
\hline 1.60 & 24.93 & 94.28 & 59.38 & -25.90 & 11.91 & 29.63 & 4.92 & 22.87 & -1.15 & -20.89 & 0.0005 & 0.01 \\
\hline 1.70 & 24.81 & 94.03 & 59.09 & -25.84 & 11.86 & 29.55 & 5.54 & 25.79 & -1.29 & -23.56 & 0.0005 & 0.01 \\
\hline 1.80 & 24.69 & 93.79 & 58.81 & -25.77 & 11.7987 & 29.48 & 6.14 & 28.67 & -1.43 & -26.18 & 0.0005 & 0.01 \\
\hline
\end{tabular}

Table 4: Identities of example transmitters for Benin City (Source [14]).

\begin{tabular}{lllllc}
\hline Site ID & City/District & Operating Frequency & Longitude & Latitude & BSC \\
\hline ED0014 & Benin & $900 \mathrm{MHz}$ & 5.6083918 & 6.3985011 & EEDBS03 \\
ED0019 & Benin & $900 \mathrm{MHz}$ & 5.6579429 & 6.2879423 & EEDBS01 \\
ED0068 & Ugbowo & $1800 \mathrm{MHz}$ & 5.6116694 & 6.3819425 & EEDBS03 \\
ED0096 & Ugbowo & $1800 \mathrm{MHz}$ & 5.6219203 & 6.4072237 & EEDBS03 \\
\hline
\end{tabular}


Table 5: RMSE and MPE metrics for QMM models calibrated with measurements from the transmitters of Table 4.

\begin{tabular}{|c|c|c|c|c|c|c|c|c|}
\hline \multirow{3}{*}{ Model/Metric } & \multicolumn{8}{|c|}{ Locations } \\
\hline & \multicolumn{2}{|c|}{ ED0014 } & \multicolumn{2}{|c|}{ ED0019 } & \multicolumn{2}{|c|}{ ED0068 } & \multicolumn{2}{|c|}{ ED0096 } \\
\hline & RMSE & MPE & RMSE & MPE & RMSE & MPE & RMSE & MPE \\
\hline COST231(RUR)-QMM & 1.824 & 0.339 & 3.058 & -0.353 & 2.867 & -0.054 & 2.554 & -0.049 \\
\hline COST231 (S-URB) QMM & 1.804 & -0.212 & 3.045 & -0.217 & 3.561 & 2.112 & 3.164 & 1.884 \\
\hline ECC (MEDIUM)-QMM & 0.770 & -0.022 & 0.993 & -0.021 & 1.460 & 0.055 & 0.631 & -0.048 \\
\hline ECC (LARGE) -QMM & 0.770 & -0.038 & 0.933 & -0.039 & 1.464 & 0.123 & 0.640 & 0.117 \\
\hline ERICSSON QMM & 3.860 & 3.477 & 4.633 & 3.498 & 2.867 & -0.054 & 2.554 & -0.047 \\
\hline
\end{tabular}
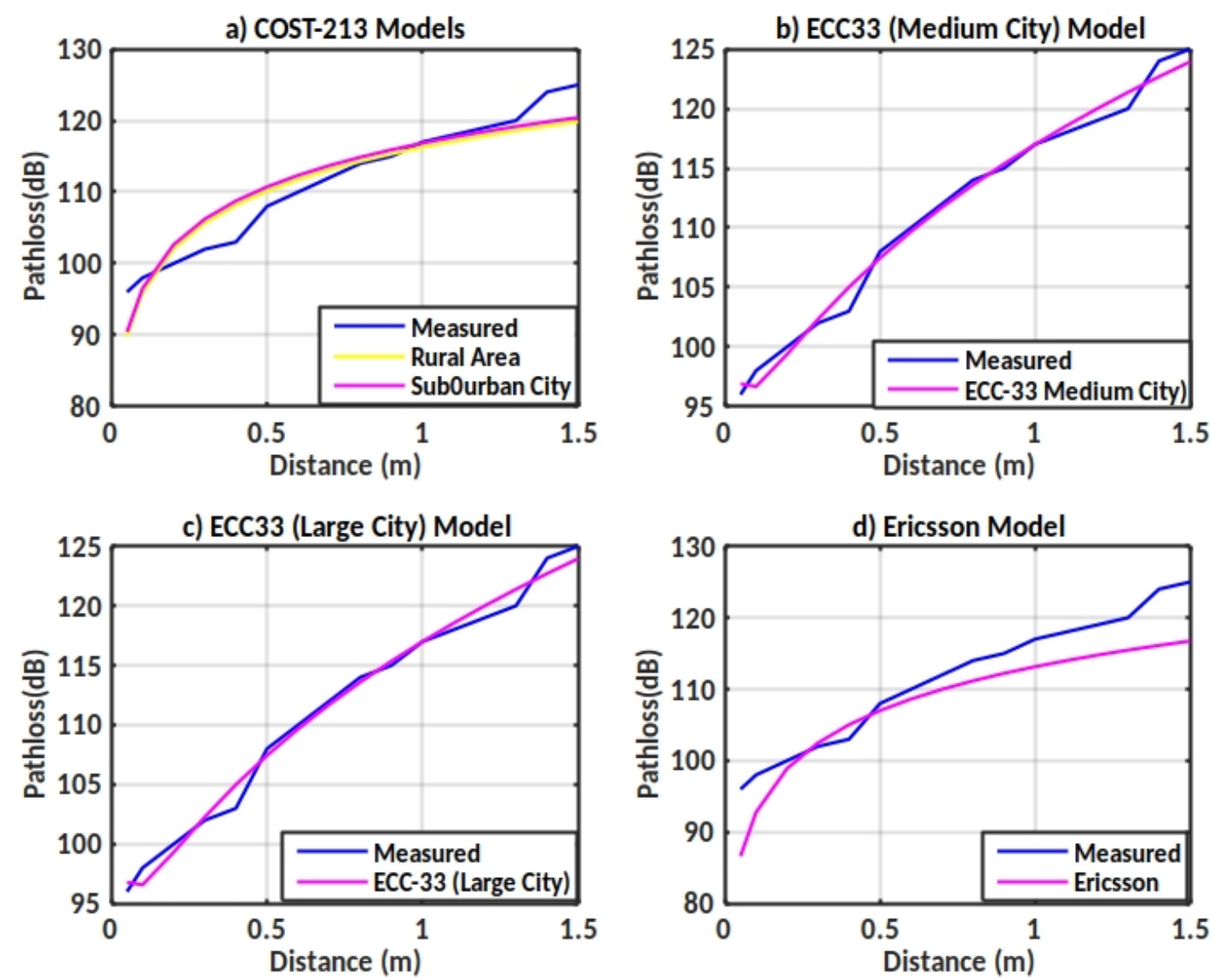

Figure 6: Comparison of pathloss predicted by QMM-models with measured data (ED0096). 

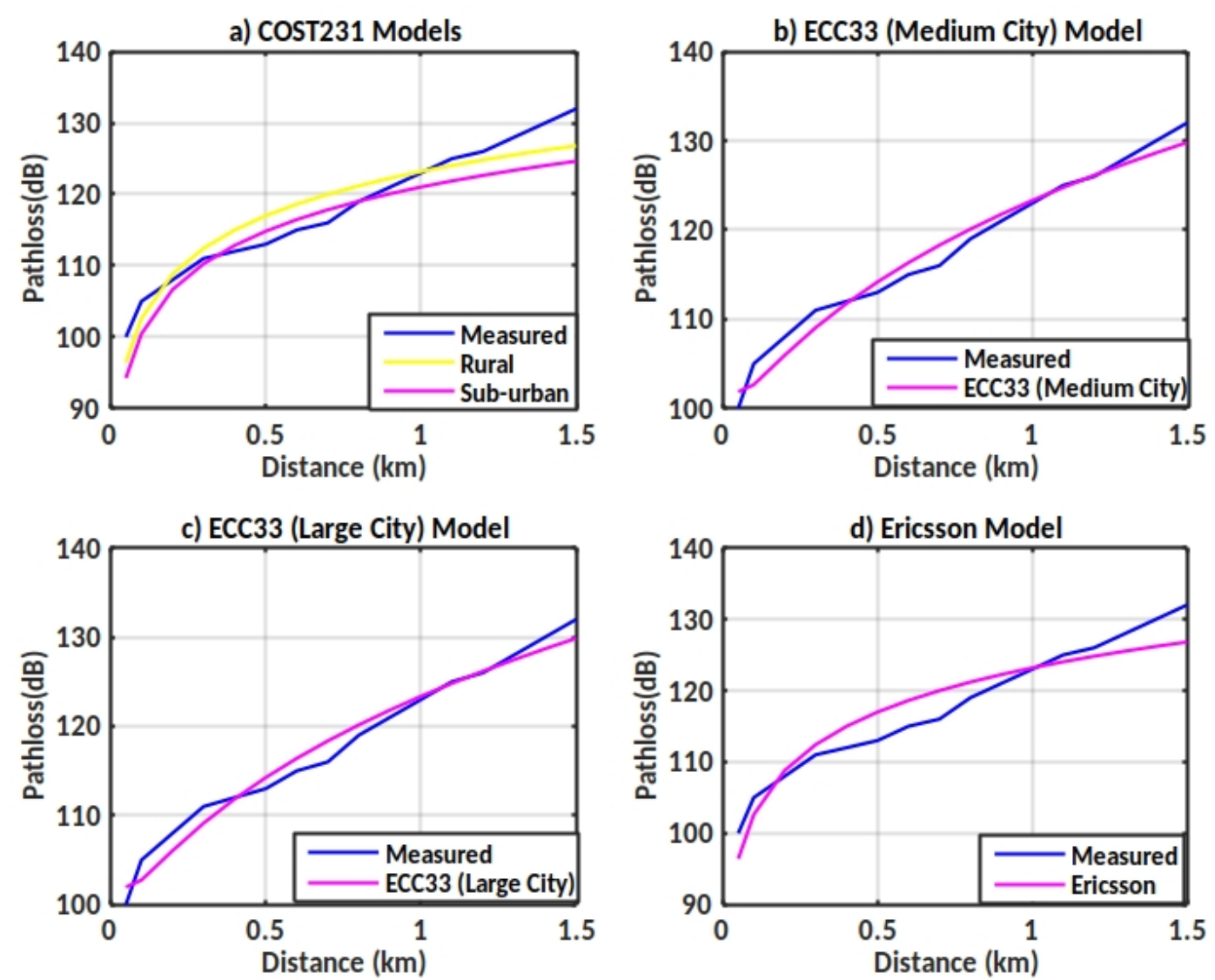

Figure 7: Comparison of pathloss predicted by QMM-models with measured data (ED0014).
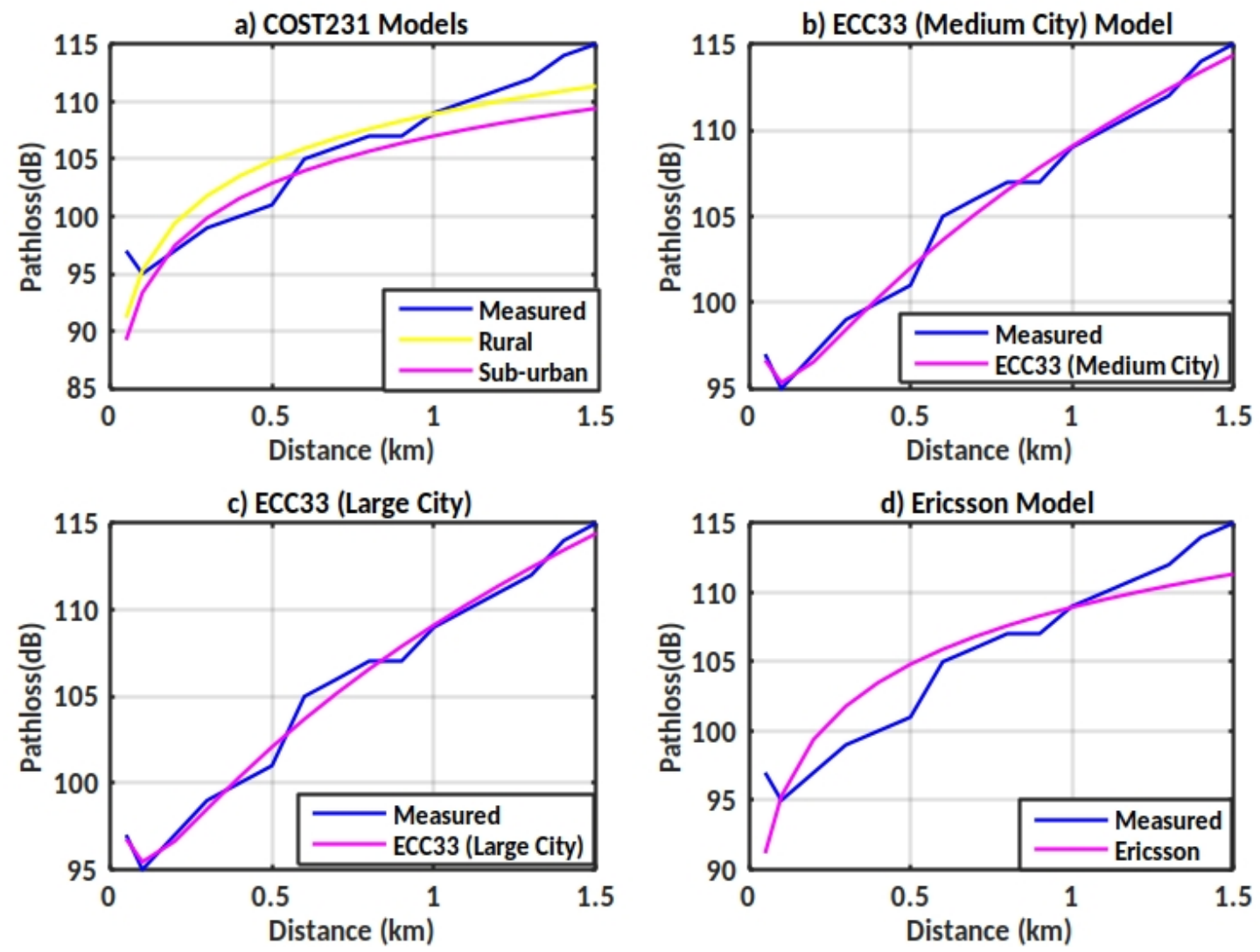

Figure 8: Comparison of pathloss predicted by QMM-models with measured data (ED0019). 
recorded RMSE values of $0.77 \mathrm{~dB}$, though the MPE of $-0.022 \mathrm{~dB}$ recorded by the medium city model is slightly better than the $-0.038 \mathrm{~dB}$ for the large city model. This pattern is closely followed by the metrics for the $1800 \mathrm{MHz}$ network with respective RMSE values of $1.460 \mathrm{~dB}$ and $1.464 \mathrm{~dB}$ for the medium and large cities, for which MPE was recorded as $0.055 \mathrm{~dB}$ and $0.123 \mathrm{~dB}$, respectively. Both calibrated COST231 models performed significantly better than the calibrated Ericsson model, with the calibrated model for rural areas being the better of the two COST231 models.

\section{CONCLUDING REMARKS}

An evaluation of the Quasi-Moment-Method (QMM) as a radiowave propagation pathloss modeling tool for use with the Benin City environment, has been undertaken in this paper. A brief description of the characterizing features of the QMM was followed by model validation, through prediction performance comparisons with results available in a recent publication in the open literature. The comparisons reveal that all the five candidate models, after QMM-calibration, performed better than the 'optimum' model reported by the publication alluded to in the foregoing. Results of QMM-calibration using field measurement data acquired for certain representative areas of Benin City by authors, confirmed the suggestion by the results of the validation exercise, that for Benin City, QMM-calibrated ECC-33 models are best suited for pathloss prediction.

One important conclusion arising from the investigations reported in this paper is that whereas results reported in the open literature suggest that the basic COST231-Hata prediction model, which typically provides the error metrics in most outdoor radiowave propagation environments $[7,15,16]$, is consequently the best candidate for model performance optimization schemes, QMM calibration has shown that this is not necessarily the case. Indeed, the results in this paper reveal that the basic ECC-33 with the worst error metrics compared to the other basic models, performs best, after QMM-calibration.

It is readily verified that in all cases, QMM's 'model-calibration matrix' is a square, symmetric matrix, characterized by a dominant eigenvalue. This suggest the possibility of the formulation of an eigenvalue problem, through which characteristic modes can be specified, and possibly, insightful physical interpretations obtained: that possibility is under investigations.

Finally, it is apparent that the QMM, as comprehensively described and utilized in this paper, represents an excellent candidate for investigations concerning the 'cross-application' of pathloss prediction models, as proposed by Zhang et al. [17].

\section{ACKNOWLEDGEMENT}

The authors gratefully acknowledge the critiques of two anonymous reviewers, which informed the significant revision of an earlier version of the paper.

\section{References}

[1] J. Chebil, A. Lwas, M. Islam, and A. Zyoud, "Adjustment of Lee Path Loss Model for Suburban Area in Kuala Lumpur-Malaysia," in Proc. of CSIT IACSIT, vol. 5, Singapore, 2011, pp. 252-257.

[2] C. Dalela, M. Prasad, and P. Dalela, "Tuning of cost231 hata model for radio wave propagation predictions," Computer Science \& Information Technology, pp. 255267, 2012.

[3] K. Diawuo, K. Dotche, and T. Cumberbatch, "Data Fitting to Propagation Model Using Least Square Algorithm: A Case Study in Ghana," International Journal of Engineering Sciences, vol. 2, no. 6, pp. 226-230, June 2012 .

[4] A. Deme, D. Dajab, and D. Nyap, "Computer Analysis of the COST 231 Hata Model and Least Squares Approximation for Path Loss Estimation at $900 \mathrm{MHz}$ on the Mountain Terrains of the Jos-Plateau, Nigeria," Computer Engineering and Intelligent Systems, vol. 4, no. 9, pp. 39-48, 2013

[5] A. Salami, K. Dotche, A. Ajavon, and K. Sa-Bedja, "Propagation Models Calibration in Mobile Cellular Networks: A Case Study in Togo," in Proc. Future Technologies Conference (FTC), Vancouver, Canada, November 2017, pp. 923-927.

[6] P. Keawbunsong, S. Duangsuwan, P. Supanakoon, and S. Promwong, "Quantitative Measurement of Path Loss Model Adaptation Using the Least Squares Method in an Urban DVB-T2 System," International Journal of Antennas and Propagation, 2018.

[7] E. Omoze and F. Edeko, "Statistical Tuning of COST 231 Hata Model In Deployed 1800MHz GSM Networks for a Rural Environment," Nigerian Journal of Technology, vol. 39, no. 4, pp. 1216-1222, 2020.

[8] G. Dahquist and A. Bjorck, Numerical Methods, N. Anderson, Ed. New York: Dover Publishers Inc. Mineola, 1974.

[9] R. Harrington, "Matrix methods for field problems," in Proceedings of the IEEE, vol. 55, no. 2, 1967, pp. 136149.

[10] $\stackrel{149 .}{-}$ Field Computation by Moment Methods. New York: The Macmillan Company, 1968.

[11] L. Kantrovich and V. Krylov, Approximate Methods of Higher Analysis. New York: John Wiley \& Sons Inc. 1959.

[12] J. Milanovic, S. Rimac-Drlje, and K. Bejuk, "Comparison of Propagation Models Accuracy for WiMAX on $3.5 \mathrm{GHz}$, in 14th IEEE International Conference on Electronics, Circuits and Systems, Marrakech, 2007, pp. 111-114.

[13] A. Bostan and P. Dumas, "Wronskians and linear independence," The American Mathematical Monthly, vol. 117 , no. 8, pp. 722-727, January 2010.

[14] M. Adelabu, "Optimum propagation pathloss prediction model for the nigerian mobile radio communication environment," Ph.D. dissertation, School of Postgraduate Studies, University of Lagos, Akoka, Lagos, Nigeria, 2016.

[15] U. Anamonye, G. Efenedo, and M. Okuma, "Evaluation and analysis of gsm signals in warri," Nigerian Journal of Technology (NIJOTECH), vol. 35, no. 2, pp. 568-578, April 2016.

[16] A. Obot, O. Simeon, and J. Afolayan, "Comparative analysis of path loss prediction models for urban macro cellular environments," Nigerian Journal of Technology, vol. 30, no. 3, pp. 50-59, October 2011.

[17] J. Zhang, C. Gentile, and W. Garey, "n the crossapplication of calibrated pathloss models using area features: finding a way to determine similarity between areas," in IEEE Antennas and Propagation Magazine, vol. 62 , no. 1, February 2020 , pp. 40-50. 